\title{
Drug Use Changes at the Individual Level: Results from a Longitudinal, Multisite Survey in Young Europeans Frequenting the Nightlife Scene
}

\author{
Meryem Grabski ${ }^{a}$ Jon Waldron ${ }^{a} \quad$ Tom P. Freeman $^{\mathrm{a}, \mathrm{b}} \quad$ Claire Mokrysz $^{\mathrm{a}}$ \\ Ruben J.J. van Beek ${ }^{c}$ Peggy van der Pol ${ }^{d}$ Bert Hauspie $^{e} \quad$ Nicky Dirkx $^{e}$ \\ Jochen Schrooten $^{f}$ Tobias H. Elgán ${ }^{g}$ Kristin Feltman ${ }^{g}$ Elisa Benedetti ${ }^{\text {h }}$ \\ Gianpaolo Scalia Tombai ${ }^{i}{\text { Francesco Fabi }{ }^{j} \text { Sabrina Molinaro }}^{\text {h }}$ \\ Johanna Gripenberg ${ }^{g}$ Tina van Havere ${ }^{e}$ Margriet van Laar ${ }^{c} \quad H$. Valerie Curran $^{a}$ \\ ${ }^{a}$ Clinical Psychopharmacology Unit, University College London, London, UK; ${ }^{b}$ Addiction and Mental Health Group \\ (AIM), Department of Psychology, University of Bath, Bath, UK; 'Trimbos Institute, The Netherlands Institute of \\ Mental Health and Addiction, Utrecht, The Netherlands; ${ }^{d}$ NETQ Healthcare (Utrecht), Topicus Healthcare Company

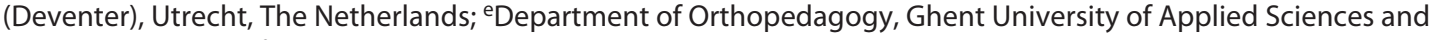 \\ Arts, Gent, Belgium; 'VAD (Vereniging voor Alcohol en andere Drugproblemen), Brussels, Belgium; ${ }^{9}$ STAD, Centre for \\ Psychiatry Research, Department of Clinical Neuroscience, Karolinska Institutet, \& Stockholm Health Care Services, \\ Region Stockholm, Stockholm, Sweden; ' Institute of Clinical Physiology, National Research Council, Pisa, Italy; \\ 'Department of Mathematics, University of Rome Tor Vergata, Rome, Italy; 'Centro Studi Statistici e Sociali (Ce3S), \\ Pisa, Italy
}

\section{Keywords}

Longitudinal online survey · Drug use $\cdot$ Ketamine $\cdot$ Novel psychoactives · Prescription opioids · Nightlife scene

\begin{abstract}
Background: Monitoring emerging trends in the increasingly dynamic European drug market is vital; however, information on change at the individual level is scarce. In the current study, we investigated changes in drug use over 12 months in European nightlife attendees. Method: In this longitudinal online survey, changes in substances used, use frequency in continued users, and relative initiation of use at follow-up were assessed for 20 different substances. To take part, participants had to be aged 18-34 years; be from Belgium, Italy, the Netherlands, Sweden, or the UK; and have attended at least 6 electronic music events in the past 12 months at base-
\end{abstract}

karger@karger.com www.karger.com/ear

Karger $\frac{1}{6}$
(C) 2021 The Author(s)

Published by S. Karger AG, Basel

This article is licensed under the Creative Commons Attribution 4.0 International License (CC BY) (http://www.karger.com/Services/ OpenAccessLicense). Usage, derivative works and distribution are permitted provided that proper credit is given to the author and the original publisher. line. Of 8,045 volunteers at baseline, 2,897 completed the survey at both time points (36\% follow-up rate), in 2017 and 2018. Results: The number of people using ketamine increased by $21 \%(p<0.001)$, and logarithmized frequency of use in those continuing use increased by $15 \%(p<0.001 ; 95 \%$ Cl: $0.07-0.23)$. 4-Fluoroamphetamine use decreased by $27 \%$ $(p<0.001)$, and logarithmized frequency of use in continuing users decreased by $15 \%(p<0.001,95 \% \mathrm{Cl}:-0.48$ to -0.23$)$. The drugs with the greatest proportion of relative initiation at follow-up were synthetic cannabinoids $(73 \%, N=30)$, mephedrone $(44 \%, N=18)$, alkyl nitrites $(42 \%, N=147)$, synthetic dissociatives $(41 \%, N=15)$, and prescription opioids $(40 \%, N=48)$. Conclusions: In this European nightlife sample, ketamine was found to have the biggest increase in the past 12 months, which occurred alongside an increase in frequency of use in continuing users. The patterns of uptake and discontinuation of alkyl nitrates, novel psychoactive 
substances, and prescription opioids provide new information that has not been captured by existing cross-sectional surveys. These findings demonstrate the importance of longitudinal assessments of drug use and highlight the dynamic nature of the European drug landscape.

(c) 2021 The Author(s)

Published by S. Karger AG, Basel

\section{Introduction}

In the increasingly dynamic European drug landscape, the close monitoring of emerging trends is vital to anticipate and react to changes. Country-specific differences ranging from policy frameworks to data collection methods provide a unique challenge for the assessment of drug use in Europe. The European Monitoring Centre for Drugs and Drug Addiction (EMCDDA) responds to this challenge by pooling national data, police statistics, and methods such as wastewater analysis in their yearly report [1]. On an international level, the Global Drug Survey assesses a wide array of drug use behaviors yearly [2]. However, currently there are few longitudinal, pan-European data that can adequately capture shifts of drug use patterns at the individual level.

The Electronic Music Scene Survey (EMSS) is a longitudinal online survey assessing nightlife and drug use behavior in young adults regularly attending nightlife events in Belgium, Italy, the Netherlands, Sweden, and the UK. Investigations of harder to reach populations that are more likely to use illicit substances, like young adults in the nightlife scene, can be used to anticipate drug use trends that may later manifest in the general population, adding value to patterns of use found in population surveys [3-5].

In the current study, we used the following measures from the Electronic Music Scene Survey (EMSS) in order to assess how changes in the past 12-month use and frequency of use measured on the individual level compare to cross-sectional drug use data: (i) 12-month drug use trajectories, (ii) frequency of drug use trajectories in continuing users, and (iii) initiation of use (i.e., first lifetime use at follow-up) of 20 different substances.

\section{Methods}

Survey participants had to reside in one of the 5 participating countries, be between 18 and 34 years old, and to have attended at least 6 dance/electronic music events during the past 12 months. Participants were recruited mostly online using convenience sampling through Facebook and Instagram advertisements. A valida- tion study found that this online sample was broadly representative of the target population recruited from clubs and festivals [6].

Completers of the baseline survey were invited for the followup survey after 12 months via email, the only contact information stored (May-November 2018). Baseline participants were included in a lottery to win electronic gadgets (6 Macbooks, 6 I-pads, and 15 Bluetooth speakers), and those that completed the follow-up survey received a guaranteed reimbursement (GBP 20/EUR 20 Amazon voucher).

The EMSS investigated different aspects of nightlife and drug use behaviors (see online suppl. Table S1; for all online suppl. material, see www.karger.com/doi/10.1159/000520118). The following items of the EMSS will be discussed here: at baseline, ever use of a list of 21 drugs was assessed with the item "Have you used any of the following drugs in your lifetime"?; past 12-month use was assessed both at baseline and follow-up with the item "Have you used this drug in the past 12 months"?; frequency of use was assessed both at baseline and follow-up with the item "How often did you use this drug in the last 12 months"?, with the response options "3 times a week or more" [6], "weekly" [5], "fortnightly" [4], "monthly" [3], "every 2 or 3 months" [2], and " 3 times or less in the year" [1]. All drugs assessed in the survey were investigated here (with the exception of heroin due to its rare use): alcohol, amphetamines, benzodiazepines, cannabis, cocaine, N,N-dimethyltryptamine, ecstasy, 4-fluoroamphetamine (4-FA), gamma hydroxybutyrate, heroin, ketamine, lysergic acid diethylamide, magic mushrooms, mephedrone, nitrous oxide, alkyl nitrite ("poppers"), prescription opioids, synthetic cannabinoids, synthetic dissociatives, synthetic hallucinogens, and tobacco.

\section{Analysis}

The baseline survey had 8,045 completers and the follow-up 2,897 ( $36 \%$ follow-up rate). Only responses of participants that completed both baseline and follow-up were included in this study $(N=2,897)$.

Attrition was investigated by comparing baseline demographics and the past 12-month substance use of follow-up completers $(N=2,897)$ with noncompleters $(N=$ $5,148)$ using $\chi^{2}$ tests. Percentage change for use of each drug is described by the ratio of difference between past 12-month use at follow-up and past 12-month use at baseline to the past 12-month baseline use.

Relative initiation at follow-up for each drug is described by the ratio of participants indicating 12-month use at follow-up only to the overall 12-month use of this drug at follow-up. This was done to standardize incidence rates against drug-specific prevalence, which was considered more meaningful in this context than calculating the ratio of initiation of 1 specific drug to the overall sample. Twelve months of use at follow-up only comprised participants indicating never use at baseline, no past 12-month use at baseline, and past 12-month use at follow-up. 


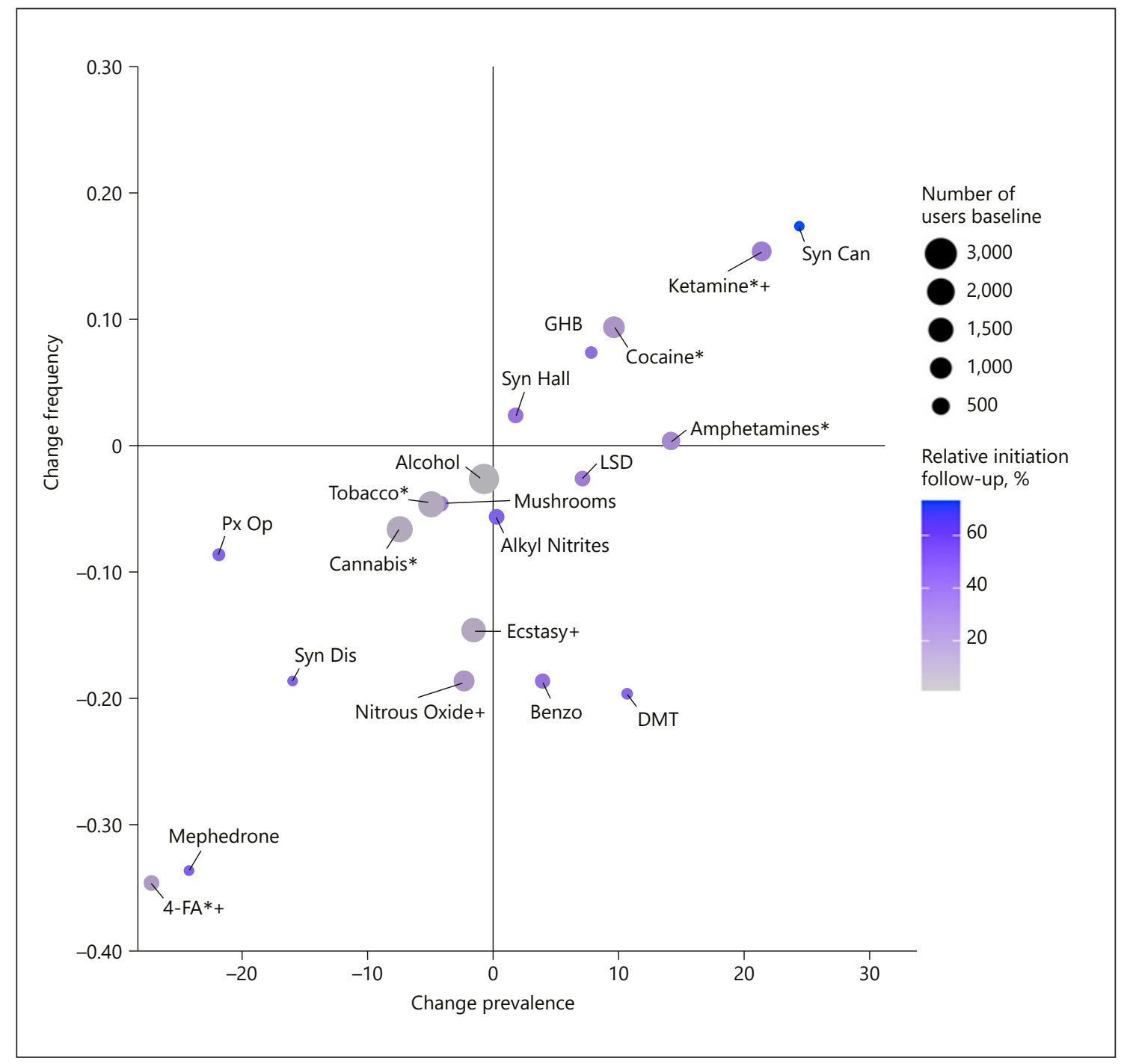

Fig. 1. The relationship of changes in the past 12-month use ("prevalence"), changes in the past 12-month logarithmized frequency of use ("frequency"), and relative initiation of use at followup (e.g., first ever use). The size of each dot indicates the amount of past 12-month users at baseline (range: 33-2,785), and the color indicates the percentage of novel use at follow-up (range: $1.12 \%-$ $73.12 \%)$. ${ }^{*}$ Change in baseline prevalence Bonferroni adjusted
$(0.05 / 20=0.0025) ;{ }^{+}$change in logarithmized frequency Bonferroni adjusted $(0.05 / 20=0.0025)$. DMT, N,N-dimethyltryptamine; 4-FA, 4-fluoroamphetamine; Benzo, benzodiazepines; GHB, gamma hydroxybutyrate; LSD, lysergic acid diethylamide; Px Op, prescription opioids; Syn Can, synthetic cannabinoids; Syn Dis, synthetic dissociatives; Syn Hall, synthetic hallucinogens.
The past 12-month frequency of use was converted into days of use per year. Days of use per year were then log-transformed for analysis due to the skewed distribution of the variable.

Changes in drug use at baseline and follow-up were analyzed using McNemar's $\chi^{2}$ test, and for the calculation of odds ratios and associated 95\% confidence intervals, the McNemar exact test was used [7]. Changes in logarithmized frequency of drug use at baseline and follow- up were analyzed using dependent-samples $t$ tests. Changes in logarithmized frequency were only assessed in participants who indicated use at both time points, referred to as "continued users." All $p$ values described were Bonferroni adjusted to account for multiple testing. All analyses were conducted using R statistical software (Foundation for Statistical Computing, Vienna, Austria). 


\section{Results}

\section{Attrition}

A comparison of follow-up completers and noncompleters at baseline revealed a lower percentage of male than female follow-up completers, a greater number of Dutch follow-up completers, and fewer Italian follow-up completers compared to baseline completers. Significant differences in drug use were found for tobacco, which follow-up completers were less likely to use, and 4-FA, which was much higher in follow-up completers (online suppl. Table S1).

\section{Demographics}

The mean age at baseline was 23.8 (SD 4.46) years, and the sample was predominantly male (66.4\%). Residence at baseline assessment was distributed as follows: $17.09 \%$ of participants resided in Belgium, $11.77 \%$ in Italy, $29.00 \%$ in the Netherlands, $17.19 \%$ in Sweden, and $24.96 \%$ in the UK.

\section{Drug Use}

The relationship of drug use frequency and prevalence, as well as the relative initiation for all drugs in the sample, is displayed in Figure 1. All results reported below are statistically significant. The most widely used drugs at both time points were alcohol ( $96 \%$ at baseline [BL]), tobacco (68\% BL), cannabis (62\% BL), and ecstasy (54\% BL) (see online suppl. Table S3). The greatest increase in the past 12 -month use was recorded for ketamine from 744 at BL to 902 at $\mathrm{FU}\left(21 \%, \chi^{2}[1]=58.41\right.$, OR $2.20,95 \%$ CI: $1.78-$ 2.72), amphetamines from 675 at BL to 770 at $\mathrm{FU}\left(14 \%, \chi^{2}\right.$ $[1]=22.48$, OR 1.64, 95\% CI: 1.33-2.02), and cocaine from 1,055 at $\mathrm{BL}$ to 1,156 at $\mathrm{FU}\left(10 \%, \chi^{2}[1]=23.41, \mathrm{OR} 1.62,95 \%\right.$ CI: 1.33-1.98) (see online suppl. Table S3). On the country level, this increase was biggest in the Netherlands for all 3 drugs (see online suppl. Table S4). The greatest significant decrease was for 4-FA from 333 at BL to 243 at FU (-27\%, $\chi^{2}[1]=38.83$, OR 0.39, 95\% CI: 0.28-0.53), cannabis from 1805 at BL to 1,672 at $\mathrm{FU}\left(-7 \%, \chi^{2}[1]=33.19\right.$, OR $0.60,95 \%$ CI: $0.50-0.71$ ), and tobacco from 1971 at BL to 1,874 at FU $\left(-5 \%, \chi^{2}[1]=20.17\right.$, OR 0.65, 95\% CI: 0.54-0.79) (see online suppl. Tables S2 and S3). On the country level, the decrease of 4-FA was greatest in the Netherlands (the only country with considerable use), the decrease of cannabis was greatest in the UK, and the decrease of tobacco was greatest in the Netherlands (see online suppl. Table S4).

The only significant increase of logarithmized frequency in continued users was recorded for ketamine (MD 0.15, 95\% CI: 0.07-0.24). Due to the natural log transformation, this mean difference can be interpreted as a percentage change: participants increased their frequency of use by $15 \%$ between baseline and follow-up. Thus, on average, a participant using ketamine 6 times in the past 12 months at baseline would have increased their use to about 7 times in the past 12 months at follow-up. High-risk ketamine use (weekly or more) did not increase and was relatively low at both time points $(<30$ participants). The greatest significant decrease in logarithmized frequency for continued users was found for 4-FA (MD $0.15,95 \%$ CI: -0.48 to -0.23 ), and thus participants decreased their frequency of use by $15 \%$ between baseline and follow-up (online suppl. Table S5).

The greatest percentage of relative initiation at followup was recorded in synthetic cannabinoids $(73 \%, N=30)$, mephedrone ( $44 \%, N=18)$, alkyl nitrates $(42 \%, N=147)$, synthetic dissociatives $(41 \%, N=15)$, and prescription opioids $(40 \%, N=48)$ (see online suppl. Table S3). On the country level, the UK had the highest percentage of follow-up past 12-month use of synthetic cannabinoids, mephedrone, and prescription opioids and the Netherlands of alkyl nitrates and synthetic dissociatives (see online suppl. Table S4).

\section{Discussion}

Our analysis assessing changes in drug use on the individual level in a European nightlife sample has several implications. First, due to the longitudinal nature of this study, we were able to investigate individual uptake of drugs between baseline and follow-up. Furthermore, as ever use was assessed at baseline, we could establish initiation at follow-up. The drugs with the greatest proportion of relative initiation at follow-up were synthetic cannabinoids, mephedrone, alkyl nitrates, synthetic dissociatives, and prescription opioids. However, the total number of initiators for synthetic cannabinoids, mephedrone, and synthetic dissociatives was each below 30 . Interestingly, there was almost no change in the overall past 12 -month use of alkyl nitrates and a decrease in the past 12-month use of mephedrone, synthetic dissociatives, and prescription opioids. This suggests that a high proportion of participants who had indicated use of these drugs at baseline had discontinued use, and different participants had initiated use at follow-up. The infrequent use of these drugs could point toward an interest in trying them in parts of the population, as well as unexpected or unwanted effects leading to discontinuation after initial use, which could explain the high rate of discontinuation after baseline. However, there are other explanations for 
this finding such as discontinued availability, or given that the number of users of some of these substances is quite small, a subpopulation that is generally more experimental in their drug use. Overall, these findings show that cross-sectional data can mask the uptake of a drug in specific parts of the population. This is especially relevant as the risk associated with first-time use might be higher than that in other drug use occasions. Further research should investigate whether harm reduction strategies for these substances may best be directed at first-time users.

Second, measures of ketamine use showed the highest increase in the past 12 months and in logarithmized frequency of use at the individual level as well as a considerable increase in new initiates (237) at follow-up. However, high-risk ketamine use (weekly or more) did not increase. This longitudinal increase in the past 12-month ketamine use aligns with cross-sectional population surveys showing an increase in use in European countries, such as the UK and the Netherlands $[8,9]$. Here, we show that continued users also increased the frequency of use by approximately $15 \%$ at the individual level. Both initiation and escalation of drug use are potentially harmful behaviors, and further research should investigate whether potential first-time users and regular users might benefit from different harm reduction approaches. For example, potential first-time users might benefit from information promoting prevention of first use or information on minimizing risk at the first use, whereas current users might benefit from information about and strategies directed at preventing escalation of use. Amphetamines and cocaine showed a significant increase in the past 12 -month use in line with findings from the EMCDDA indicating increased use across Europe, according to population surveys as well as wastewater analyses, and an increase in seizures of these drugs [1]. Especially in the case of cocaine, this has led to a recent surge in treatment demand related to cocaine use, likely boosted by an increase in purity as well as a decrease in price in the European market [10]. In the current survey, a total of 171 participants indicated they initiated cocaine use by follow-up. Future research, including the linkage of different datasets, might allow for estimating the time span between initiation of use, increase of use, and need for treatment, potentially allowing for the prediction of future treatment seeking.

Last, the drug with the greatest decrease in both past 12-month use and logarithmized frequency of use was 4-FA. This development might be linked to health warnings followed by a ban of 4-FA in the Netherlands in 2017, where it previously had a high prevalence [11]. Our analysis shows that the frequency of use by continuing users

Drug Use Changes at the Individual Level in Europe also decreased by $15 \%$. However, as this sample was somewhat biased toward 4-FA users, these results should be interpreted with caution.

\section{Limitations}

Attrition was relatively high with only one-third of baseline participants completing the follow-up survey. Even though there were no significant differences between completers and noncompleters on most variables, several key variables showed significant differences: completers were more likely to be female, Dutch, and users of 4-FA and less likely to be Italian and tobacco smokers. Thus, even though the baseline sample has been validated against the target population at clubs and festivals, we cannot exclude the possibility of bias specifically regarding gender, nationality, 4-FA use, and smoking status in the current sample.

\section{Conclusion}

The individual uptake and discontinuation of use of alkyl nitrates, certain novel psychoactive substances, and prescription opioids over time is varying more than crosssectional data might suggest. We furthermore show that the increase of ketamine in recent years is accompanied by increases in both logarithmized frequency of use and initiation of use. These findings demonstrate the importance of longitudinal assessments of drug landscapes, particularly with regard to developing targeted harm reduction strategies and drug policy initiatives.

\section{Statement of Ethics}

The study was approved by the UCL Ethics Committee (Ethics Project ID: 10437/001). Written informed consent was collected digitally from participants before the start of the baseline survey by ticking a box.

\section{Conflict of Interest Statement}

The authors have no conflicts of interest to declare.

\section{Funding Sources}

ALAMA-nightlife is a collaborative project supported by the European Research Area Network on Illicit Drugs (ERANID). This study is based on independent research commissioned and funded in England by the National Institute for Health Research (NIHR) Policy Research Programme (project ref. PR- 
ST-0416-10003). The views expressed in this article are those of the authors and not necessarily those of the national funding agencies or ERANID.

\section{Author Contributions}

M.G. contributed to study design, analysis, interpretation, initial draft, revisions, and final approval and agrees to be accountable for all parts of the work. J.W. contributed to study design, interpretation, initial draft, revisions, and final approval and agrees to be accountable for all parts of the work. T.P.F. contributed to acquisition, interpretation, initial draft, revisions, and final approval and agrees to be accountable for all parts of the work. C.M. contributed to interpretation, revisions, and final approval and agrees to be accountable for all parts of the work. R.J.J.B. contributed to interpretation, revisions, and final approval and agrees to be accountable for all parts of the work. P.P. contributed to interpretation, revisions, and final approval and agrees to be accountable for all parts of the work. B.H. contributed to interpretation, revisions, and final approval and agrees to be accountable for all parts of the work. N.D. contributed to interpretation, revisions, and final approval and agrees to be accountable for all parts of the work. J.S. contributed to interpretation, revisions, and final approval and agrees to be accountable for all parts of the work. T.H.E. contributed to interpretation, revisions, and final approval and agrees to be accountable for all parts of the work. K.F. contributed to interpretation, revisions, and final approval and agrees to be accountable for all parts of the work. E.B. contributed to interpretation, revisions, and final approval and agrees to be accountable for all parts of the work. G.S.T. contributed to interpretation, revisions, and final approval and agrees to be accountable for all parts of the work. F.F. contributed to interpretation, revisions, and final approval and agrees to be accountable for all parts of the work. S.M. contributed to interpretation, revisions, and final approval and agrees to be accountable for all parts of the work. J.G. contributed to acquisition, interpretation, revisions, and final approval and agrees to be accountable for all parts of the work. T.H. contributed to acquisition, interpretation, revisions, and final approval and agrees to be accountable for all parts of the work. M.L. contributed to acquisition, interpretation, revisions, and final approval and agrees to be accountable for all parts of the work. H.V.C. contributed to acquisition, initial draft, interpretation, revisions, and final approval and agrees to be accountable for all parts of the work.

\section{Data Availability Statement}

The data generated or analyzed during this study are currently not publicly available; however, any enquiries can be directed to the corresponding author.

\section{References}

1 European Monitoring Centre for Drugs and Drug Addiction (EMCDDA). European drug report 2019: trends and developments. Luxembourg: Publications Office of the European Union; 2019.

2 Winstock A, Ferris J, Barratt MJ, Maier LJ. (Plenary) Global Drug Survey 2019 key findings: brief summary; 2019.

3 Ferrence R. Diffusion theory and drug use. Addiction. 2001;96(1):165-73.

4 Bretteville-Jensen AL, Burdzovic Andreas J, Gjersing L, Øiestad EL, Gjerde H. Identification and assessment of drug-user groups among nightlife attendees: self-reports, breathalyzer-tests and oral fluid drug tests. Eur Addict Res. 2019;25(2):93-102.
5 Betzler F, Ernst F, Helbig J, Viohl L, Roediger L, Meister S, et al. Substance use and prevention programs in Berlin's party scene: results of the SuPrA-study. Eur Addict Res. 2019; 25(6):283-92.

6 Waldron J, Grabski M, Freeman TP, Mokrysz C, Hindocha C, Measham F, et al. How do online and offline sampling compare in a multinational study of drug use and nightlife behaviour? Int J Drug Policy. 2020;82: 102812.

7 Fay MP. Exact McNemar's test and matching confidence intervals. 2020. Retrieved from: https://cran.microsoft.com/snapshot/2015-06-23/web/packages/exact2x2/vignettes/exactMcNemar.pdf.
8 Home office. Drug misuse: findings form the 2018/19 crime survey for England and Wales. UK: Home office; 2019.

9 Van Laar MW, Cruts AAN, Van Miltenburg CJA, Strada LV, Ketelaars APM, Croes EA, et al. Nationale drug monitor: jaarbericht 2019. Utrecht: Trimbos-instituut; 2020.

10 Antoine J, Berndt N, Astudillo M, Cairns D, Jahr S, Jones A, et al. Cocaine treatment demands in 10 western European countries: observed trends between 2011 and 2018. Addiction. 2021;116(5):1131-43.

11 Van der Pol P, Nijlkamp L, Nabben T, Van Laar M. 4-FA-4-Fluoramfetamine: Gebruikers en gebruik in beeld. 2017. 